\title{
Fast and Accurate Statistical Estimation of Common Mode Voltages and Currents in Weakly Non-Uniform Differential Interconnects
}

\author{
Xinglong Wu(1), Flavia Grassi ${ }^{(1)}$, Paolo Manfredi ${ }^{(2)}$, Giordano Spadacini ${ }^{(1)}$, Dries Vande Ginste ${ }^{(2)}$, Sergio A. Pignari ${ }^{(1)}$ \\ (1) Dept. of Electronics, Information and Bioengineering, Politecnico di Milano, Milan, Italy \\ (2) Dept. of Information Technology, IDLab, Ghent University-imec, Gent, Belgium
}

\begin{abstract}
In this work, an approximate procedure for the fast evaluation of modal voltages and currents induced at the terminations of non-ideal differential interconnects is proposed. The procedure is exploited in combination with Monte Carlo simulations, in order to efficiently obtain statistical estimates of undesired common mode voltages and currents arising from asymmetries and nonuniformities introduced by the manufacturing process. Compared to the standard solution method, the proposed procedure allows a significant reduction of computational times, while retaining the same prediction accuracy.
\end{abstract}

\section{Introduction}

In recent years, statistical approaches gained increasing attention from Electromagnetic Compatibility (EMC) engineers, due to the necessity to model structures affected by parameter uncertainty resulting from lack of knowledge and/or manufacturing tolerances. The standard (non-intrusive) approach, commonly known as Monte Carlo (MC), to tackle such a modeling issue resorts to the generation of a suitably large number of random samples of the structure under analysis, and to repeated deterministic simulations aimed at the evaluation of statistical estimates of target quantities. Therefore, since the overall model efficiency is set by the computational cost of the underlying deterministic model, the development of fast and accurate solution approaches is highly desirable in order to reduce simulation time.

In this perspective, an approximate but computationally efficient procedure to statistically analyze the effects owing to undesired asymmetries and non-uniformities affecting differential interconnects is here proposed. Such a procedure leverages the perturbation approach in [1], which is here rephrased in the modal domain and simplified under the assumption of weak imbalance [2]. The proposed approach is here exemplified by means of a canonical differential-line (DL) circuit composed of a pair of coupled microstrips with geometrical characteristics that differ from the nominal ones due to imperfection in the manufacturing process. An enhanced MoM-based solver is used for the evaluation of the corresponding perunit-length (p.u.l.) parameters, whose efficient evaluation is made critical due to the combined effect of medium inhomogeinity and line non-uniformity.

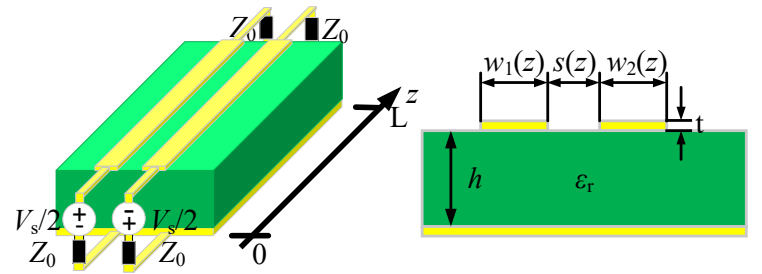

Figure 1. Principle drawing of the ideal DL under study (left) and associated PCB cross-section (right).

The proposed method allows obtaining statistical estimates of common-mode (CM) voltages and currents at the DL terminals with a significant reduction of computational costs with respect to the standard solution approach (in the following referred to as uniform cascade sections (UCS) method) [3], nevertheless retaining the same prediction accuracy.

\section{MTL modeling}

\subsection{Ideal vs stochastic DL under analysis}

A principle drawing of the ideal differential line (DL) circuit under analysis is shown in Fig. 1. The line is composed of two ideally identical traces printed on the top side of a double-sided printed-circuit board (PCB), characterized by the following geometrical/electrical data: $h=1.425 \mathrm{~mm} ; \varepsilon_{\mathrm{r}}=4.4 ; t=0.035 \mathrm{~mm} ; s=0.5 \mathrm{~mm}$; $w_{1}=w_{2}=0.6 \mathrm{~mm} ; L=150 \mathrm{~mm}$. An ideal DM source $\left(V_{s}=1\right.$ $\mathrm{V})$ drives the DL from the near-end. Additionally, terminal sections that are perfectly balanced with respect to ground as well as ideally matched to the DM impedance of the two traces (i.e., $Z_{0}=Z_{D M} / 2$ ) are connected at both line ends.

To account for possible imperfections in the manufacturing process, a perturbation of the geometrical parameters of the aforesaid ideal structure is introduced as shown in Fig. 2. Particularly, at the DL end-points (i.e., for $z=0$ and for $z=L$ ) the width of the two traces (i.e., $w_{1}(0), w_{1}(L)$ and $\left.w_{2}(0), w_{2}(L)\right)$ as well as their separation (i.e., $\mathrm{s}(0), \mathrm{s}(L))$ are treated as random variables $(\mathrm{RVs})$, with variation around their nominal values described by the normal distributions in Table 1 . The actual trace layout is then obtained by joining these random startingand end-points (keeping the trace axes fixed), thus allowing the stochastic generation of several PCB configurations to be exploited in the MC simulations. 


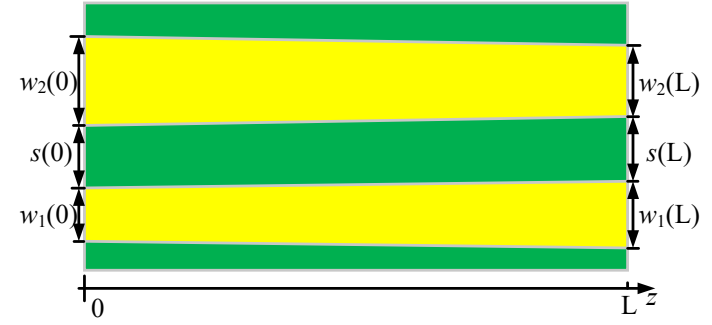

Figure 2. Non-ideal trace layout.

Table 1. Variation of trace parameters

\begin{tabular}{|c|c|}
\hline $\mathrm{RV}$ & $N\left(\mu, \sigma^{2}\right), \mathrm{mm}$ \\
\hline$w_{l}(0), w_{l}(L)$ & $\mu=0.6, \quad \sigma=0.03$ \\
\hline$w_{2}(0), w_{2}(L)$ & $\mu=0.6, \quad \sigma=0.03$ \\
\hline$s(0), s(L)$ & $\mu=0.5, \quad \sigma=0.025$ \\
\hline
\end{tabular}

\subsection{Numerical evaluation of p.u.l. parameters}

In order to get statistical estimates of the response of the non-ideal DL under analysis, the generation and analysis of several DL samples are required. However, line nonuniformity in combination with medium inhomogeneity poses issues concerning the efficient evaluation of the involved p.u.l. parameters. To speed up the p.u.l. parameter evaluation, an improvement of the MoM-based numerical solver proposed in [4] is hereinafter used. In [4], trace thickness was assumed to be infinitesimally small. In the proposed implementation instead, empirical correction factors are introduced to account for the nonnull thickness of the PCB traces. More precisely, self and mutual p.u.l. inductances and capacitances are evaluated by resorting to equivalent traces, still of infinitesimal thickness but with width and separation modified as follows

$$
\begin{gathered}
w_{e q, 1(2)}=w_{1(2)}+\alpha_{w 1(2)} \beta_{w} t, \\
s_{e q}=s-2 \alpha_{S} \beta_{S} t,
\end{gathered}
$$

where $w_{1(2)}, s$ and $t$ are the actual widths, separation, and thickness of the traces, whereas $\alpha, \beta$ are empirical correction factors for the shape and relative permittivity, respectively, with expressions:

$$
\begin{gathered}
\alpha_{w 1(2)}=\frac{w_{1(2)}+h}{w_{1(2)}+h+t}, \quad \alpha_{S}=\frac{s+h}{2(s+h+t)}, \\
\beta_{w}=2\left[\frac{\left(\varepsilon_{r}-1\right) h}{2(h+t)}+\frac{\varepsilon_{r}+1}{2}\right]^{-1}, \quad \beta_{S}=\frac{2\left(3 \varepsilon_{r}-1\right)}{\varepsilon_{r}\left(\varepsilon_{r}+1\right)} .
\end{gathered}
$$

The use of the correction factors in (3), (4) yields an appreciable increase in the accuracy of the inductance and capacitance p.u.l. parameters. This is proven in Fig. 3, where the maximum relative error, defined as the maximum between the errors on each p.u.l. parameter

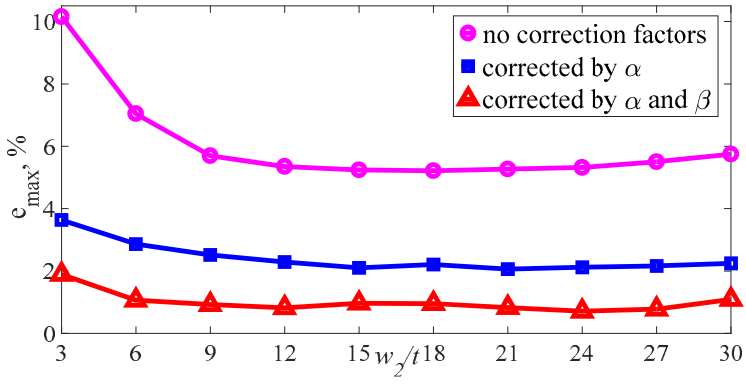

Figure 3. Improvement in the accuracy of the p.u.l. parameters achieved by modifying the actual PCB-trace dimensions by the correction factors in (3), (4).

$\ell_{i j}, c_{i j}$ with respect to the reference values $\bar{\ell}_{i j}, \bar{c}_{i j}$ calculated by Ansys-Maxwell [5], i.e.,

$$
e_{\max }=\max \left(\left|\frac{\ell_{i j}-\bar{\ell}_{i j}}{\bar{\ell}_{i j}}\right|,\left|\frac{c_{i j}-\bar{c}_{i j}}{\bar{c}_{i j}}\right|\right)_{i, j=1,2},
$$

is plotted as function of the width-to-thickness ratio $w_{2} / t$. The magenta curve is obtained by means of the original code in [4]. The blue curve is obtained by applying the correction factors in (3) only, whereas the red curve is obtained by applying both the correction factors in (3) and (4). In general, maximum discrepancies with respect to [5] are observed for the mutual p.u.l. capacitance, whose value results to be lower than the actual one if trace thickness is neglected.

\section{Approximate vs exact solution}

For the solution of the resulting non-uniform DL, an approximate approach, based on the concept of perturbation [1] and exploiting the assumption of weak imbalance [2], is here adopted with the objective to reduce the computational burden associated with the standard solution (hereinafter UCS) based on the discretization of the line into uniform subsections [3].

The proposed procedure is based on [1], where line nonuniformity is treated as a perturbation of a reference uniform line with p.u.l. parameters averaged along the line length. As shown in [6], in the absence of dielectric material, for DL circuits such an approach can be easily reformulated for DL circuits in the modal domain and simplified by resorting to the assumption of weak imbalance [2]. This leads to a significant decrease in computational time with respect to the UCS method. Furthermore, taking the CM and DM equivalent circuits associated with the ideal DL structure (i.e., the perfectly uniform and matched DL in Fig. 1) as reference-line, allows to clearly put in evidence the perturbative effect that undesired non-uniformities affecting DLs introduce on the modal voltages and currents.

Indeed, in the modal domain the assumption of weak 


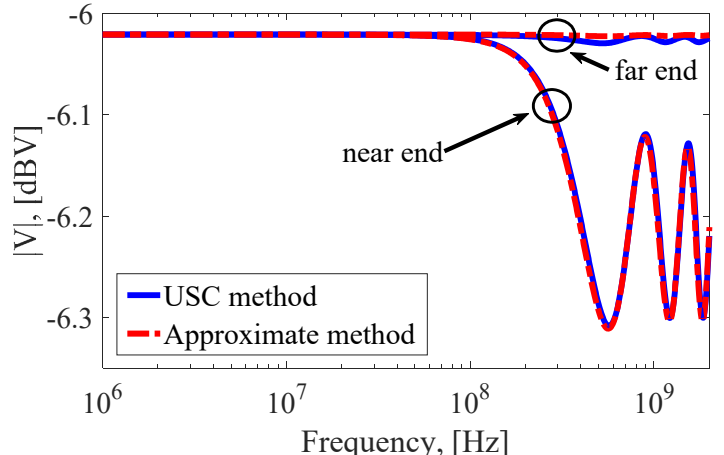

(a)

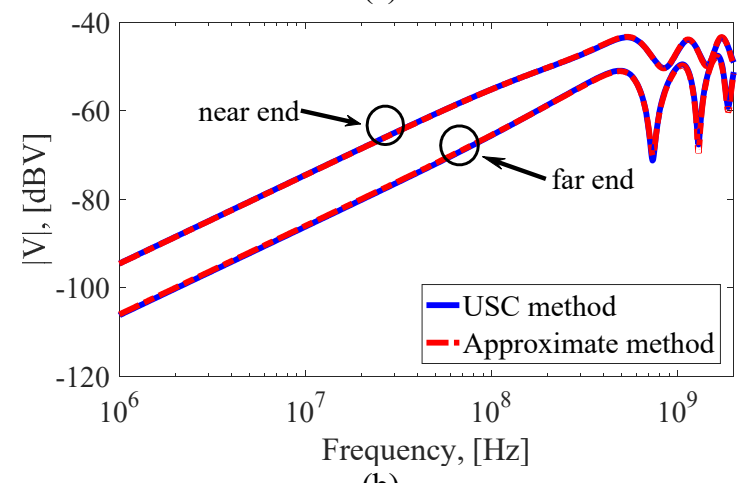

(b)

Figure 4. Comparison of (a) DM and (b) $\mathrm{CM}$ voltages at line terminals as predicted by the UCS model and by the proposed approximate procedure.

imbalance allows approximating the involved Telegrapher Equations [6] as

$$
\begin{aligned}
& \left\{\begin{array}{l}
\frac{d V_{D M}(z)}{d z}+j \omega \tilde{\ell}_{D M} I_{D M}(z) \cong-j \omega \Delta \ell_{D M}(z) I_{D M}(z), \\
\frac{d I_{D M}(z)}{d z}+j \omega \tilde{c}_{D M} V_{D M}(z) \cong-j \omega \Delta c_{D M}(z) V_{D M}(z),
\end{array}\right. \\
& \left\{\begin{array}{l}
\frac{d V_{C M}(z)}{d z}+j \omega \tilde{\ell}_{C M} I_{C M}(z) \cong-j \omega \Delta \ell(z) I_{D M}(z), \\
\frac{d I_{C M}(z)}{d z}+j \omega \tilde{c}_{C M} V_{C M}(z) \cong-j \omega \Delta c(z) V_{D M}(z),
\end{array}\right.
\end{aligned}
$$

where: $\tilde{\ell}_{D M}, \tilde{c}_{D M}, \tilde{\ell}_{C M}, \tilde{c}_{C M}$ are the DM and CM p.u.l. parameters of the ideal structure; $\Delta \ell, \Delta c$ are the offdiagonal entries of the modal p.u.l. matrices of the actual DL; and $\Delta \ell_{D M}, \Delta c_{D M}$ denote the difference between the p.u.l. DM inductance and capacitance of the actual and ideal DL.

Since the assumption of weak imbalance allows neglecting the back-interaction of the CM onto the DM, equations in (6) do not depend on the CM. As a consequence, DM quantities can be computed as the first step, by iterative solution [1] of an equivalent twoconductor TL characterized by (a) the DM p.u.l. parameters of the ideal DL, and (b) equivalent distributed sources proportional to DM voltages and currents evaluated at the previous iteration step. The solution is then cast as a sum of perturbations, e.g., $V_{D M} \cong \tilde{V}_{D M}+V_{D M}^{(1)}+\ldots V_{D M}^{(n)}$, where $n$ denotes the total number of iterations. Once the DM quantities have been refined, the $\mathrm{CM}$ quantities are evaluated in a single step only, by including into the ideal CM circuit distributed voltage and current sources proportional to the calculated DM quantities.

As a trade-off between computational efficiency and prediction accuracy, in the remainder of this work the order of iteration for the DM will be set to $n=1$. The accuracy achieved by this approximate solution is shown in Fig. 4, where the predictions of modal voltages at the line terminals are compared with those obtained by the UCS model for a specific DL sample with: $w_{1}(0)=0.61$ $\mathrm{mm}, w_{1}(L)=0.69 \mathrm{~mm}, w_{2}(0)=0.65 \mathrm{~mm}, w_{2}(\mathrm{~L})=0.55$ $\mathrm{mm}, s(0)=0.45 \mathrm{~mm}, s(L)=0.55 \mathrm{~mm}$.

\section{Statistical analysis}

For the statistical analysis, 1000 samples of the stochastic micro-strip DL in Fig. 2 were generated, according to the intervals of variation of the six RVs indicated in Table I. For each configuration, the p.u.l. parameters were evaluated at 200 points along $z$. MC simulations, aimed at the prediction of modal voltages at line terminals, were run by using both the UCS and the approximate method introduced in Section 3. Explicative results are shown in Fig. 5, where the obtained CM voltages (thin curves) at the left DL terminal are plotted in the frequency interval from $1 \mathrm{MHz}$ up to $2 \mathrm{GHz}$ along with relevant statistical estimates (solid-blue curves for the UCS method, dashedred curves for the approximate one). The approximate model allowed a twenty-time reduction of computational costs. Namely, the repeated simulations through the USC method required approximately 33715 s, whereas only $1519 \mathrm{~s}$ were required by the approximate one (on a PC Intel(R) Core(TM) i5-4210U, CPU running at $1.7 \mathrm{GHz}$ and 4 GB of RAM). Furthermore, these simulations proved that prediction accuracy is not appreciably affected, although the proposed approach neglects the back-interaction of the $\mathrm{CM}$ on the $\mathrm{DM}$ and uses here a single refinement for the $\mathrm{DM}$ (i.e., $V_{D M} \cong \tilde{V}_{D M}+V_{D M}^{(1)}$ ).

In spite of the relatively small range of variation of the geometrical data, $\mathrm{MC}$ simulations reveal a significant spread of the CM voltages with a confidence interval $( \pm \sigma)$ of around $20 \mathrm{~dB}$ around the mean value, and a difference on the order of $55 \mathrm{~dB}$ between the $0.5 \%$ and $99.5 \%$ percentiles. Furthermore, the simulation results in Fig. 5 allows identifying two separate frequency regions characterized by a different frequency response of the $\mathrm{CM}$ voltages. Indeed, in the low frequency region, the obtained curves on average exhibit a linearly increasing frequency response with $+20 \mathrm{~dB} /$ decade slope. Conversely, a complex frequency response characterizes 


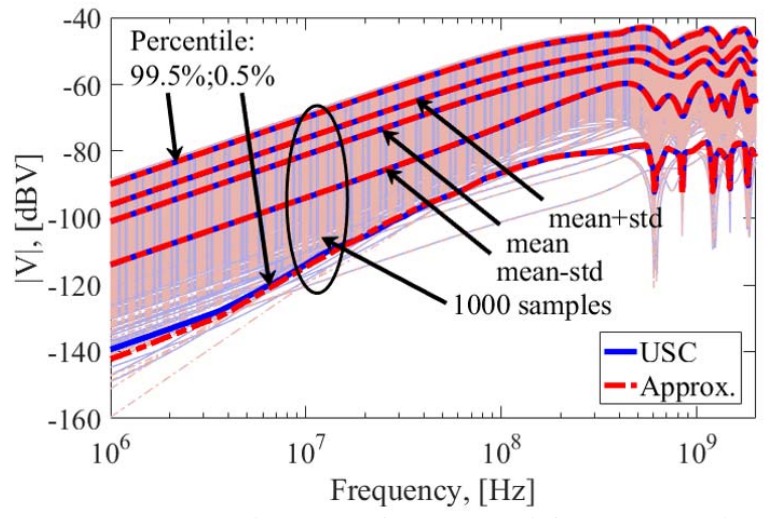

Figure 5. CM voltages at the near-end for 1000 random DL configurations and relevant statistical estimates.

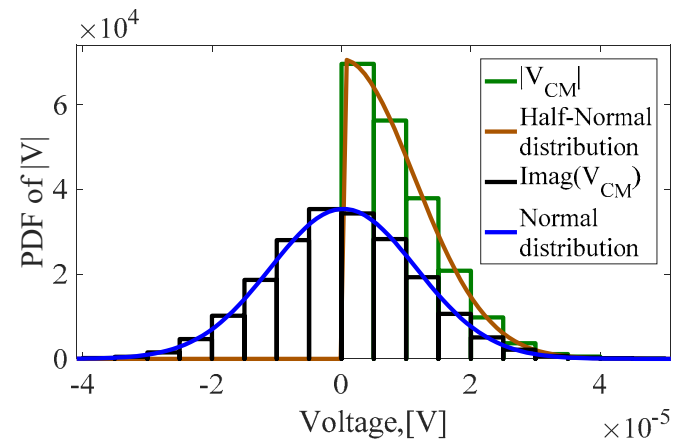

(a)

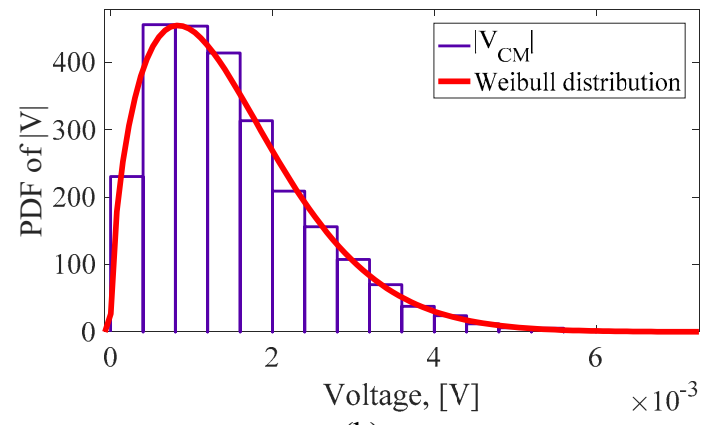

(b)

Figure 6. Probability distribution functions of the nearend $\mathrm{CM}$ voltage at (a) $1 \mathrm{MHz}$ and (b) $2 \mathrm{GHz}(5000$ samples).

the high frequency part of the spectrum (standing-wave region).

For each one of the two regions, a specific frequency was selected (i.e., $1 \mathrm{MHz}$ and $2 \mathrm{GHz}$, respectively), and 5000 repeated simulations were run by exploiting the proposed procedure in order to investigate the probability distribution functions (pdf) of near-end CM voltages. The obtained histograms are shown in Fig. 6(a) and Fig. 6(b), respectively.

At low frequency, the obtained histogram (green histogram in Fig. 6(a) is well fitted by a half-normal distribution (brown curve in Fig. 6(a)) with mean $\mu \cong 9$ $\mu \mathrm{V}$ and variance $\sigma \cong 6.8 \mu \mathrm{V}$. Such a result can be interpreted in the light of the fact that at low frequencies the CM voltage is a purely imaginary quantity. Therefore, the mean value and variance of the half-normal distribution depend on those of the corresponding normal distribution (blue curve in Fig. 6(a)) obtained by fitting the histogram of the imaginary part of the near-end CM voltage at $1 \mathrm{MHz}$ (black histogram in Fig. 6(a)). Conversely, the histogram at $2 \mathrm{GHz}$ is appreciably fitted by a Weibull distribution with $\mu \cong 1.48 \mathrm{mV}$ and $\sigma \cong 1 \mathrm{mV}$. Kolmogorov-Smirnov tests (with 5\% significance level) confirmed the goodness of fit of these distributions.

\section{Conclusions}

In this work, an approximate procedure for the fast evaluation of modal voltages and currents at the terminations of differential interconnects with a crosssection that is affected by asymmetries and nonuniformities was presented and validated versus the standard solution based on line subdivision into uniform sections [3]. The procedure is based on the perturbation approach in [1], and exploits the assumption of weak imbalance to simplify the involved Telegrapher equations. It was proven by an application example that the proposed procedure allows a twenty-time reduction of the computational costs, thus being significantly more suitable than the standard one [3] when statistical analysis through repeated $\mathrm{MC}$ simulations is the target.

\section{References}

1. P. Manfredi, D. De Zutter, D. Vande Ginste, "Analysis of nonuniform transmission lines with an iterative and adaptive perturbation technique," IEEE Trans. Electromagn. Compat., 58, 3, June 2016, pp. 859-867, doi: 10.1109/TEMC.2016.2523604.

2. F. Grassi, Y. Yang, X. Wu, G. Spadacini, S. A. Pignari, "On mode conversion in geometrically unbalanced differential lines and its analogy with crosstalk," IEEE Trans. Electromagn. Compat., 57, 2, Apr. 2015, pp. 283291, doi: 10.1109/TEMC.2014.2372894.

3. M. Omid, Y. Kami, M. Haya "Field coupling to nonuniform and uniform transmission lines," IEEE Trans. Electromagn. Compat., 39, 3, Aug. 1997, pp. 201-211, doi: $10.1109 / 15.618047$.

4. C. R. Paul, Analysis of Multiconductor Transmission Lines, Wiley Interscience, NY: New York, 1994.

5. Ansys Maxwell SV. Available online at http://www.ansys.com/products/electronics/ansysmaxwell

6. F. Grassi, P. Manfredi, X. Liu, J. Sun, D. Vande Ginste, S. A. Pignari, "Effects of undesired asymmetries and nonuniformities in differential lines," submitted to IEEE Trans. Electromagn. Compat. 